\title{
Efectos adversos a nivel cardiovascular y neurológico asociados al consumo de modafinilo
}

\author{
Diego Felipe Sanabria Lozano* \\ Nefi Helaman Rodriguez Londoño* \\ Dikson Jair Logreira Gonzalez* \\ Andrea Marquez Barbosa* \\ Orlando Jose Mejia Restrepo*
}

\begin{abstract}
*Estudiante X nivel de Medicina. Facultad de Salud. Universidad De Santander-Udes. Bucaramanga. Santander. Colombia. Correspondencia: Sr Nefi Rodriguez. Dirección: Calle 105 número 42a-09. Barrio San Bernardo. Floridablanca. Santander. Colombia. Número telefónico:3045967722. Correo electrónico: Nefi0831@gmail.com
\end{abstract}

Resumen

Introducción: el modafinilo es un fármaco neuroestimulante utilizado principalmente para promover estados de vigilia atención y disminuir la fatiga ante ciertos comportamientos que propician la somnolencia diurna excesiva. Objetivo: identificar en la literatura científica los efectos adversos neurológicos y cardiovasculares causados por el consumo del modafinilo. Materiales y Métodos: revisión bibliográfica de los artículos encontrados entre los meses de abril y julio de 2019 en las bases de datos PUBMED, SCOPUS, DIALNET. 51 artículos superaron la evaluación de calidad metodológica y se incluyeron en la revisión. Resultados: se identificaron que los principales efectos adversos a nivel cardiovascular son la cardiomiopatía Tako-Tsubo y la taquicardia ventricular polimórfica, mientras que a nivel neurológico puede generar insomnio y distonías. Conclusiones: El consumo del modafinilo genera repercusiones en las funciones cognitivas y cardiovasculares por lo cual no es aconsejable su uso a largo plazo en personas sanas. MÉD. UIS.2020;33(1):31-8.

Palabras claves: Taquicardia ventricular. Distonía. Modafinilo. Estimulantes del sistema nervioso central .Trastornos del Sueño-Vigilia. Narcolepsia.

\section{Adverse effects at the cardiovascular and neurological level associated with the consumption of modafinil}

Abstract

Introduction: modafinil is a neurostimulant drug used mainly to promote wakefulness, attention and decrease fatigue in certain behaviors that cause excessive daytime sleepiness. Objective: identify in the scientific literature the neurological and cardiovascular adverse effects caused by the consumption of modafinil. Materials and Methods: bibliographic review of the articles found between the months of April and July of 2019 in the PUBMED, SCOPUS, DIALNET databases. 51 articles passed the methodological quality assessment and were included in the review. Results: the main adverse effects at the cardiovascular level were identified as Tako-Tsubo cardiomyopathy and polymorphic ventricular tachycardia, while at the neurological level it can generate insomnia and dystonia. Conclusions: the consumption of modafinil generates repercussions on cognitive and cardiovascular functions, so its long-term use in healthy people is not advisable. MÉD.UIS.2020;33(1):31-8.

Keywords: Ventricular tachycardia. Dystonia. Modafinil. Central nervous system stimulants. Sleep-wake disorders. Narcolepsy

¿Cómo citar este artículo?: Sanabria DF, Rodríguez NH, Logreira DJ, Marquez A, Mejía OJ. Efectos adversos a nivel cardiovascular y neurológico asociados al consumo de modafinilo. MÉD. UIS.2020;33(1):31-8. doi: 10.18273/revmed.v33n1-2020004 


\section{Introducción}

El modafinilo es un fármaco que fue aprobado para venta libre por primera vez en Francia en el año 1992 y posteriormente en Estados Unidos desde 1998 por la Food and Drug Administration. Inicialmente fue lanzado para promover la vigilia y atención ante ciertos estados de comportamiento que propician la somnolencia excesiva diurna, ya sea por patologías clínicas en adultos, e.g. narcolepsia comprobada con o sin cataplejía, hipersomnia idiopática, apnea obstructiva del sueño o por trabajos en turno nocturno (personal del área de salud, del ejército, pilotos, azafatas, conductores, etc) que conllevan a trastornos de regulación sueño-vigilia',2.

El modafinilo se ha destacado como un neuroestimulante pro-vigilia que disminuye la fatiga, sin embargo, su uso se ha ampliado en los últimos años en pacientes con síndrome de fatiga crónica o que padecen enfermedades como la esclerosis múltiple, el Alzheimer, mal de Parkinson, cáncer, procesos de rehabilitación de alguna enfermedad en estado crítico, déficit de atención e hiperactividad en adultos-niños y de manera experimental en pacientes con dependencia a la cocaína o a los psicoestimulantes como la metanfetamina3,4. En Latinoamérica hay pocos estudios estadísticos amplios sobre el uso no médico de psicotrópicos cognitivos. A pesar de que la información es limitada, indica un uso bastante común comparable con la evidencia internacional. La mayoría de investigaciones se focalizan en el consumo de drogas lícitas e ilícitas por parte de estudiantes, sin preguntar acerca del modafinilo y metilfenidato. El metilfenidato es un derivado de las anfetaminas que tiene el potencial de crear hábito o favorecer el desarrollo de otras adicciones Según una revisión de cuatro bases de datos realizada por la Escuela de Medicina de la Universidad de Caxias do Sul de Brasil entre 1990 y 2012, fueron seleccionados artículos en inglés, portugués y español que cumplieran los criterios establecidos acerca del uso de metilfenidato entre estudiantes de medicina, donde la prevalencia alcanzó el 16\% sin distinción entre género ${ }^{5}$. En Colombia, según el estudio nacional de consumo de sustancias psicoactivas realizado en el año 2013, se estableció que el consumo sin prescripción médica de estimulantes como el metilfenidato y modafinilo fueron muy bajos, por lo que no se consideró relevante hacer un análisis detallado para otras variables sociodemográficas ${ }^{6}$.
El efecto del modafinilo es etiquetado según creencia popular como un potenciador cognitivo o "píldora de la inteligencia" que mejora las habilidades mentales al proporcionar estados de concentración y memoria sobresalientes, lo cual ha despertado especial interés entre estudiantes universitarios, que pretenden aumentar su rendimiento académico y concentración a través del consumo de este fármaco. Sin embargo, los efectos del modafinilo en la función cognitiva han llevado a interpretaciones mixtas o contradictorias, en tanto la manifestación de reacciones adversas en adultos y niños ha generado preocupación frente a su consumo a largo plazo, razón por la cual los estudios y comisiones de investigación acerca del balance real entre su eficacia como neuroestimulante y los riesgos asociados a su consumo han aumentado en los últimos años?.

La dosis diaria de modafinilo como principio activo no debe superar los $400 \mathrm{mg}$, siendo un fármaco dopaminérgico clasificado como psicoestimulante, pero que presenta ventajas respecto a los estimulantes tradicionales, como lo son su acción relativamente rápida y de larga duración (pico plasmático de 2 a 4 h después de la ingesta con vida media de 12 a $15 \mathrm{~h}$ ), aplicando su acción a un área específica ${ }^{8}$ A diferencia de los estimulantes del sistema nervioso central, el modafinilo actúa como agente simpaticomimético de acción central inhibiendo principalmente los transportadores de catecolaminas, bloqueando específicamente la recaptación de los neurotransmisores dopamina y noradrenalina en la corteza prefrontal y ganglios basales, elevando así sus concentraciones a nivel extracelular y actuando indirectamente sin estimular los receptores adrenérgicos, ya que su efecto pro-vigilia puede ser bloqueado administrando antagonistas del receptor a1 y $\beta$-adrenérgico9. A nivel neurológico, sus efectos en la función cognitiva han sido estudiados principalmente en modelos experimentales animales con resultados mixtos que serán el foco de esta revisión de tema junto con los efectos a nivel cardiovascular. Con base en los resultados obtenidos con modelos clínicos se ha evidenciado un aumento de la actividad adrenérgica, histamínica, a nivel de la hipocretina y el sistema glutamatérgico, en cuanto disminuye la actividad del neurotransmisor inhibitorio en el sistema nervioso central GABA, en áreas específicas del cerebro (cuerpo estriado y globo pálido), respecto al hipocampo se ha detectado solo a dosis altas a 
través de un sistema serotoninérgico, reduciendo así los efectos negativos del sueño sobre la memoria en la regulación de los neurotransmisores durante un ciclo vigilia-sueño $0^{10,11,12}$. Sin embargo, debido a los usos aún no aprobados del modafinilo en especial por la aparición de casos reportados de disfunciones cardiacas y psiquiátricas transitorias en adultos, así como reacciones cutáneas y de hipersensibilidad en niños, en los últimos años se continua estudiando los efectos que pueden presentar estos fármacos en modelos no clínicos y en humanos a nivel neurológico y cardiovascular. De este modo, esta revisión de tema se enfoca en identificar los efectos colaterales en la función cognitiva y cardiovascular reportados para el modafinilo.

\section{Materiales y métodos}

La pregunta de investigación abordada fue: ¿Cuáles han sido los resultados encontrados respecto a los efectos adversos a nivel neurológico y cardiovascular asociados al uso de modafinilo?

Este problema de investigación fue tratado aplicando la metodología del sistema PICO (Population, Intervention, Comparison, Outcome), en la cual se esclarecen los siguientes elementos principales:

Población: pacientes tratados con modafinilo.

Intervención: los efectos del fármaco a nivel de la corteza cerebral y respuestas procognitivas al realizar tareas a partir de la evaluación de comportamientos, así como efectos adversos a corto y largo plazo a nivel cardiovascular y neurológico.

Comparación: con o sin grupo control (placebo).

Outcome (resultados de interés): saber cuáles son los efectos clínicos/farmacológicos de los sujetos de estudio, así como sus efectos adversos a nivel neurológico y cardiovascular a corto y largo plazo.

La revisión bibliográfica de artículos fue efectuada entre abril y julio de 2019 en las bases de datos Pubmed, Scopus y Dialnet. Habiendo sido encontrados textos desde el año 2000 hasta el año actual 2019. Los criterios de inclusión considerados fueron aquellos estudios enfocados en los efectos generados por el uso de modafinilo y las reacciones adversas observadas tras su consumo, especialmente aquellas relacionadas con patologías neurológicas y cardiovasculares, otro criterio de inclusión fue la disponibilidad de los artículos completos en las bases de datos en línea, los cuales en su mayoría están en inglés y algunos en español. Los criterios de exclusión fueron aquellos artículos por duplicidad y contenido. Se tuvieron en cuenta estos criterios con el propósito de tener la más óptima y pertinente información respecto al modafinilo. Inicialmente los artículos encontrados y la selección de ellos dependieron que sus títulos estuvieran relacionados con "modafinil cardiovascular effects", "modafinil neurological effects", "modafinil and health risks" y "modafinilo psicoestimulante". Para la metodología de elegibilidad de los artículos, inicialmente se utilizaron las palabras claves y se escogieron en las bases de datos los artículos con los títulos que más se relacionaran con los términos de búsqueda, en seguida se seleccionaron los resúmenes de mayor interés que tuvieran relación con el efecto secundario a nivel cardiovascular o neurológico que generara el modafinilo, se eligieron los artículos con base al texto completo y finalmente, para complementar la revisión se siguió la declaración Preferred Reporting Items for Systematic Reviews and MetaAnalyses, mediante la cual se consigue identificar y elegir los estudios basados en métodos explícitos y sistemáticos que permitirán validar una revisión en base a ciertos criterios de calidad e inclusión, parámetros importantes a la hora de seleccionar y sintetizar los estudios de interés con el fin de minimizar el sesgo y lograr conclusiones confiables.

\section{Resultados}

La Figura 1 muestra la organización de la revisión de tema de acuerdo a los resultados obtenidos (Ver Figura 1).

Figura 1. Esquema del proceso de inclusión de la revisión de tema.

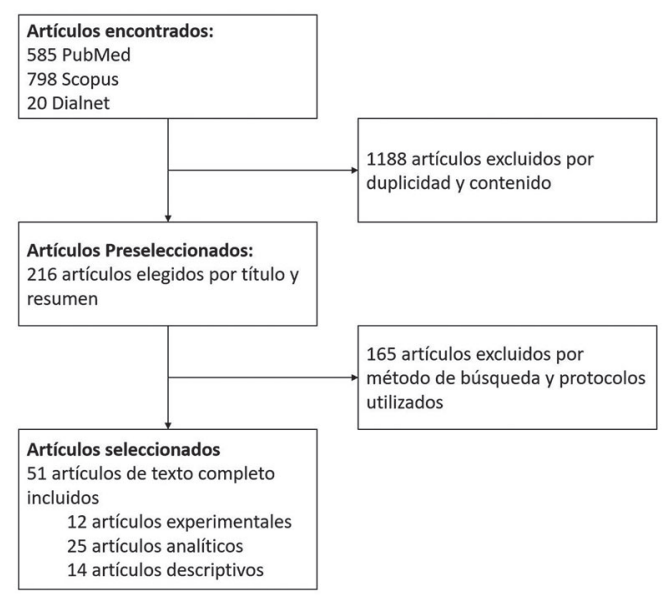

Fuente: autores. 
Entre los artículos seleccionados se tuvieron en cuenta los riesgos y el uso terapéutico del modafinilo en términos éticos y de seguridad. En los estudios analíticos y experimentales se consideró solo aquellos resultados con valores $p<0,05$, lo cual indica un posible sesgo que radica en solo la selección de artículos estadísticamente significativos.

\section{Modafinilo: seguridad y efectos adversos comunes}

Uno de los primeros estudios sobre modafinilo fue de tipo multicéntrico, aleatorizado con una muestra de 248 pacientes entre 6 y 17 años; se evaluó su eficacia y los efectos adversos producidos en niños y adolescentes con déficit de atención. El consumo en dosis diarias entre 170-425 mg mostró un bajo potencial de abuso y mejoría en el $48 \%$ de los pacientes tratados comparado con un $17 \%$ de mejoría en pacientes tratados con placebo. Efectos adversos como el insomnio (29\%), cefalea (20\%) y pérdida del apetito (16\%) fueron observados durante 9 semanas y planteó la necesidad de analizarlos a largo plazo ${ }^{13}$. En años posteriores se expusieron los efectos adversos a corto plazo (12 semanas) ${ }^{14}$ y toxicidad a largo plazo (8 años) del modafinilo en dosis diarias de 200-400 mg para pacientes adultos con somnolencia excesiva ${ }^{15}$. En el corto plazo los eventos adversos más comunes fueron: cefaleas (34\%) y náuseas (11\%), siendo además el insomnio y la pérdida de apetito los efectos secundarios más frecuentes en niños y adolescentes con déficit de atención y habiendo sido poco frecuentes los eventos adversos graves (18 casos entre 961 pacientes tratados, mientras 9 casos fueron reportados en pacientes con placebo) ${ }^{16}$. Este estudio incluyó una evaluación de la presión arterial/frecuencia cardíaca, intervalos de electrocardiograma, polisomnografía y exámenes clínicos de laboratorio. Un aumento significativo de la presión diastólica fue el efecto adverso grave más reportado; mientras que el aumento de la presión cardiaca, el sueño evaluado en la polisomnografía junto con las anomalías en el electrocardiograma fueron eventos adversos raros. Sin embargo, el beneficio asociado al consumo de modafinilo en los casos de trastornos del sueño fue más destacado que los riesgos presentados a corto plazo debido a su consumo según este estudio. A largo plazo los eventos adversos más frecuentes se asocian con taquicardia, agitación, insomnio, ansiedad, dolor de pecho, además de la elevación de creatina fosfoquinasa y presencia de distonías como efecto clínico (Ver Tabla 1). Además, aunque el modafinilo no ha mostrado niveles altos de abuso o toxicidad, despierta preocupación la escasa evidencia de pruebas clínicas que justifiquen su uso en terapias en niños con déficit de atención o como potenciador cognitivo en adolescentes sanos ${ }^{16,17}$.

Tabla 1: Efectos adversos y beneficios del consumo de modafinilo.

\begin{tabular}{|l|l|}
\hline \multicolumn{1}{|c|}{$\begin{array}{c}\text { EFECTOS ADVERSOS A CORTO } \\
\text { PLAZO }\end{array}$} & \multicolumn{1}{c|}{ EFECTOS BENÉFICOS } \\
\hline Cefalea & Trastornos del sueño \\
\hline Náuseas & $\begin{array}{l}\text { Mejora el estado de alerta, } \\
\text { vigilia y atención }\end{array}$ \\
\hline Insomnio & $\begin{array}{l}\text { Disminuye la fatiga y } \\
\text { somnolencia en el día }\end{array}$ \\
\hline Inapetencia & \\
\hline $\begin{array}{l}\text { Aumento de la presión arterial } \\
\text { diastólica }\end{array}$ & \\
\hline Comportamientos eufóricos & \\
\hline \multicolumn{2}{|c|}{ EFECTOS ADVERSOS A LARGo PLAZO } \\
\hline Comportamientos psicóticos & \\
\hline Taquicardia & \\
\hline Agitación & \\
\hline Ansiedad & \\
\hline Dolor de pecho & \\
\hline Distonías & \\
\hline Fuente: & \\
\hline
\end{tabular}

Fuente: autores.

\section{Efectos cardiovasculares}

Al igual que un gran número de otros medicamentos neuropsiquiátricos, el consumo de modafinilo está contraindicado en pacientes con cardiopatías debido ala inhibición dela recaptación de catecolaminas en las neuronas presinápticas, lo cual favorece el desarrollo de anormalidades transitorias como el aturdimiento miocárdico por catecolamina, caracterizado por el movimiento de la pared ventricular izquierda. Este evento de insuficiencia cardiaca es conocido como cardiomiopatía Tako-Tsubo o Síndrome del corazón roto por estrés (aneurisma ventricular izquierdo agudo); estudios posteriores como el elaborado por Aytas O. y Dilek H. en 2015, evidenciaron los riesgos específicos a nivel cardiovascular debido al consumo de modafinilo, siendo la cardiomiopatía TakoTsubo $^{18}$ y la taquicardia ventricular polimórfica ${ }^{19}$, considerados como casos aislados derivados de la administración de fármacos simpáticamente 
activos, como epinefrina y dobutamina ${ }^{20}$; ya que en un estudio aleatorio controlado con placebo los pacientes no presentaron aumento significativo de la frecuencia cardiaca usando bajas dosis de modafinilo (100 mg), por lo cual para pacientes con síndrome de taquicardia postural no generó eventos graves. La tolerancia al fármaco a largo plazo aún debe ser estudiada en estos casos de frecuencia cardiaca elevada con deterioro cognitivo ${ }^{21}$. Para dosis mayores y a corto plazo se realizó una comparación entre consumidores de modafinilo (400 mg) y pacientes tratados con placebo durante 3 días. Las diferencias observadas en los pacientes tratados con el fármaco fueron: aumento de la frecuencia cardiaca en reposo, aumento de la presión arterial sistólica en reposo y aumento de la presión arterial diastólica en reposo, además de un aumento ortostático de $42 \%$ en la norepinefrina plasmática, de $33 \%$ en la noradrenalina en orina y de $81 \%$ en la epinefrina en orina. De este modo, el estudio resalta que la actividad simpática (microneurografía peroneal) estuvo atenuada por el fármaco y se mantuvo la función a1-adrenoreceptora. A la luz de estos resultados clínicos se evidencia que el modafinilo debe ser contraindicado en pacientes con enfermedades cardiovasculares o que realicen ejercicio intenso regularmente, lo anterior debido a la actividad adrenérgica causada por este fármaco ${ }^{22}$. En su estudio cruzado aleatorizado, doble ciego, realizado en 2005, Taneja I. demostró mayores aumentos de la frecuencia cardíaca en pacientes jóvenes físicamente activos que ingirieron modafinilo antes de realizar ejercicios de calentamiento (134 \pm 11 vs. $119 \pm 14$ latidos por minuto), además experimentaron insomnio y un mejor rendimiento cognitivo al realizar tareas que requerían un esfuerzo mental prolongado, siendo opuesto el efecto al realizar esfuerzos mentales cortos ${ }^{23}$.

Recientemente fue realizado un estudio riguroso de cohorte basado en el análisis de información encontrada en bases de datos sobre los riesgos potenciales a nivel cardiovascular, asociados al consumo de modafinilo entre los años 2006 a 2008 en Estados Unidos. Entre las dos cohortes estudiadas (pacientes tratados por apnea obstructiva del sueño y pacientes sin medicación) se evaluaron los eventos desencadenados tras un incidente hospitalario, para lo cual se analizó desde un infarto de miocardio y un accidente cerebrovascular, la evaluación de tasas de incidencia, estimaciones de riesgo de desarrollar un accidente cardiovascular y análisis de supervivencia (regresión de Cox). Para un total de 175.524 historias clínicas de pacientes, la relación causal del modafinilo con un accidente cerebro vascular o infarto agudo al miocardio en dicha cohorte, fue de bajo riesgo en comparación con los pacientes que no consumieron modafinilo ${ }^{24}$. En otro estudio de cohorte retrospectivo se evidenció que el uso de modafinilo (170 mg) para mejorar el deterioro cognitivo de pacientes en unidad de cuidados intensivos no produjo eventos adversos graves a corto plazo (2 días), pero tampoco mostró resultados positivos en la función cognitiva durante este mismo lapso ${ }^{25}$.

\section{Efectos a nivel neurológico}

Los estudios pioneros del modafinilo en la activación neuronal en ratas por Mo $\mathrm{Y}$, Thomas $\mathrm{M}$, y Miano $\mathrm{T}$ fueron concluyentes sobre el efecto inductor de la vigilia usando ensayos de inmunorreactividad de $\mathrm{Fos}^{26}$. Hallazgos subsecuentes relacionaron el despertar característico del consumo de modafinilo con la inhibición tónica de noradrenalina en las neuronas promotoras del sueño del núcleo preóptico ventrolateral ${ }^{27}$. Intensifica la actividad tálamocortical, así como el acoplamiento eléctrico interneuronal cortical y nervioso en el núcleo olivar inferior ${ }^{28}$. Además, en dosis moderadas de hasta 300 mg su efecto no logra alterar el ritmo circadiano en roedores, cuando se administra en las noches, siendo efectivo para mejorar la vigilia a mediodía sin afectar actividades motoras ${ }^{29}$.

A nivel del núcleo accumbens hay un aumento de dopamina extracelular que puede favorecer el riesgo de adicción ${ }^{30}$; sin embargo, dada su selectividad y menor potencia como agente dopaminérgico, su acción es exclusiva a sus interacciones con el transportador de dopamina, por lo que aún no se ha comprobado su potencial de abuso, ni su efecto secundario sobre el potencial cognitivo tras la pérdida de sueño $0^{31,32,33}$. Aunque los resultados han sido positivos en lo que respecta a su uso en el tratamiento de personas adictas a la anfetamina34, también se ha comprobado que afecta la respuesta del sistema de recompensa en el núcleo accumbens en personas sanas, lo cual fue determinado al medir niveles de oxigenación analizando imágenes de resonancia magnética nuclear funcional en comparación con la condición placebo35. Además, hubo mejoras en paradigmas de aprendizaje de la memoria en ratas tras medidas comparativas indirectas entre los niveles de transportadores de dopamina en complejos fosforilados y no fosforilados (pDAT-CC y DAT-CC) con niveles de complejos conteniendo subunidades del receptor de dopamina D1- $3^{36}$. 
Por otra parte, estudios paralelos realizados con 160 estudiantes sanos no atribuyen efectos cognitivos significativos al consumir este fármaco, esto a partir de los resultados obtenidos a través de tres pruebas de aprendizaje, donde los voluntarios que consumieron modafinilo solamente se destacaron en la prueba de Stroop relacionada con la atención en comparación con el grupo placebo ${ }^{37}$. Estos resultados concordaron con un estudio posterior de casos y controles, el cual analizó por separado paradigmas de aprendizaje sobre tareas de recompensa y evasión de castigos previos (retroalimentación). Usando una dosis única de $200 \mathrm{mg}$ de modafinilo se indujo un comportamiento de elección alterado, el cual debía estar basado en la retroalimentación y no implicó una mejora cognitiva, ya que al comparar con el grupo placebo, el grupo que consumió modafinilo presentó una mayor preferencia a tomar decisiones basadas en la recompensa sin tener en cuenta la evasión de castigos $^{38}$. Este comportamiento en detrimento de la memoria y el trabajo espacial ya se había asociado al consumo de este fármaco en dosis diarias de 100 a $200 \mathrm{mg}$, de modo que su efecto cognitivo solo ha sido comprobado por cambios en la atención y vigilia (insomnio), sin mostrar mejoras durante tareas de toma de decisiones, procesamiento visual rápido o planificación espacial ${ }^{39}$. No obstante, en personas dependientes de metanfetamina y personas con deterioro cognitivo leve o demencia senil en edad temprana ${ }^{40}$, el modafinilo mejoró el rendimiento cognitivo; no ocurrió lo mismo en participantes sanos al visualizar y analizar la activación cerebral en la corteza prefrontal de la ínsula bilateral/ ventrolateral y cortezas cinguladas anteriores ${ }^{41}$. En contraste, pacientes jóvenes y sanos con privación del sueño consiguieron recuperar las fuentes delta y alfa cortical posterior de los ritmos encefalográficos cerrados gracias al modafinilo (100 mg) ${ }^{42}$.

Por otro lado, según se midió a través de pruebas neuropsicológicas no verbales en pacientes sanos sin falta de sueño, se consiguió mejorar la motivación y el rendimiento al realizar tareas de memoria, planificación y trabajo espacial, a pesar de que los resultados obtenidos usando pruebas asociadas al pensamiento creativo no fueron consistentes estadísticamente ${ }^{43}$. Una alternativa para pacientes con trastornos neuropsiquiátricos o déficit de atención fue estudiada por Gilleen y et al., quienes sugieren combinar un tratamiento farmacológico con un entrenamiento cognitivo en sesiones repetitivas. Siguiendo este principio se evaluaron los resultados de pruebas múltiples realizadas en pacientes sanos luego de consumir modafinilo (200 mg) y recibir entrenamientos diarios por 2 semanas. Durante los primeros 5 días se observó mayor agilidad en los voluntarios a la hora de aprender nuevos lenguajes, mientras que en las tareas de memoria se continuó observando un efecto nulo después de los tratamientos ${ }^{44}$. De hecho, algunos estudios que han abordado el efecto neuroestimulante de este fármaco sobre personas sanas y no privadas de sueño, percibieron que la falta de metodologías y pruebas psicométricas diseñadas específicamente para detectar efectos de mejora cognitiva en personas saludables ha generado resultados divergentes e imprecisos a nivel neurológico tras el consumo de modafinilo. De modo general, se resalta la necesidad de desarrollar nuevas metodologías para evaluar los efectos neuroestimulantes de un fármaco en pacientes sanos, lo cual demanda el diseño de pruebas más complejas para explorar diferentes dominios y subdominios neurológicos. Sin embargo, se destaca el efecto rápido y prolongado, así como el bajo riesgo respecto a los efectos secundarios o de abuso que proporciona la administración de modafinilo para aumentar la atención de personas sanas o con deficiencias cognitivas durante sesiones de entrenamiento cognitivo ${ }^{45,46}$.

Estudios posteriores como el de Battleday R. que consistió en una revisión sistemática de literatura desde enero de 1990 hasta diciembre de 2014, encontró que se aplicaron una secuencia de pruebas neuropsicológicas automatizadas usando escalas analógicas (pruebas CANTAB) antes y después del consumo de modafinilo (200 mg), mediante la cual se observó un aumento de la memoria episódica y el rendimiento de la memoria de trabajo en pacientes con depresión remitida, un trastorno que se asocia con dificultades cognitivas prolongadas ${ }^{47}$. Esta misma batería de pruebas demostró mejorías en el proceso de aprendizaje tras el consumo de modafinilo en pacientes con esquizofrenia temprana en tratamiento con antipsicóticos, así como también en el procesamiento visual rápido y la memoria verbal en pacientes sanos ${ }^{48}$. Otros pacientes con psicosis en etapa temprana mostraron mejoría en la cognición social, como el reconocimiento de expresiones faciales, es un déficit característico de la esquizofrenia, logrando evitar mediante la activación con modafinilo a través de efectos serotonérgicos en la amígdala49. Al aplicar otra secuencia de pruebas (batería cognitiva de consenso MATRICS-MCCB) a la misma población con esquizofrenia se obtuvieron resultados negativos para este fármaco. Por tanto, 
la selección previa de las pruebas psicométricas adecuadas parauna deficiencia cognitiva en específico debe ser un proceso evaluado en detalle para lograr un resultado significativo estadísticamente ${ }^{50}$

\section{Conclusiones}

El modafinilo es un fármaco que mejora el estado de alerta, vigilia y atención, disminuyendo así la fatiga y somnolencia en el día. El consumo de este fármaco se ha asociado a efectos estimulantes de la función cognitiva gracias a su inhibición de noradrenalina en las neuronas promotoras del sueño del núcleo preóptico ventrolateral, entre otros; aunque diversos estudios a nivel experimental y clínico han mostrado resultados contradictorios. Paralelamente, los estudios complementarios sobre las preocupaciones existentes a nivel cardiovascular asociadas a su consumo han mostrado bajo riesgo a corto plazo usando dosis diarias de hasta $400 \mathrm{mg}$, mientras que y a largo plazo se pueden encontrar con aumento significativo de la presión diastólica con dosis entre 200 - $400 \mathrm{mg}$ entre otros efectos adversos menos frecuentes (cardiopatia de TakoTsubo, taquicardia ventricular polimórfica). Sin embargo, debe suministrarse con precaución en pacientes con enfermedades cardiovasculares o trastornos psiquiátricos, en cuyo caso el riesgo puede superar al beneficio clínico que pueda obtenerse en su administración. No se recomienda el consumo de modafinilo a largo plazo en sujetos sanos debido a que los efectos sobre las funciones cognitivas y cardiovasculares no han sido favorables. Además, se recomienda a los investigadores realizar una selección minuciosa de pruebas psicométricas adecuadas para evaluar su efecto neurológico estadísticamente, en especial en paciente jóvenes sanos, sin privación del sueño.

\section{Agradecimientos}

Dra. Sonia Carolina González Medina

\section{Conflicto de intereses}

Los autores declaran que no tienen conflicto de intereses.

\section{Referencias bibliográficas}

1. TEVA, GmbH. (2015) Professional information of Vigil $\AA_{\text {, Ulm, }}$ Germany.

2. TEVA, Pharmaceuticals Inc. (2015) Prescribing information of Provigil®, USA.

3. Radünz L, Reuter H, Andresen-Streichert H. Modafinil in forensic and clinical toxicology- case reports, analytics and literature. J Anal Toxicol. 2018; 42: 353-359. doi:10.1093/jat/bky008

4. De la Garza R, Zorick T, London E, Newton T. Evaluation of modafinil effects on cardiovascular, subjective, and reinforcing effects of methamphetamine in methamphetamine-dependent volunteers. Drug Alcohol Depend. 2010; 106:173-80. doi: 10.1016/j.drugalcdep.2009.08.0132010

5. Loewe D. metilfenidato y modafinilo: justicia igualitaria y políticas de acceso al mejoramiento cognitivo farmacológico. Acta Bioethica. 2017; I(23).

6. Gobierno Nacional de Colombia. www.odc.gov.co. [Online].; 2013. Acceso 10 de octubre de 2019. Disponible en: https:// www.unodc.org/documents/colombia/2014/Julio/Estudio_de_ Consumo_UNODC.pdf

7. CONACE: Boletín Biblioteca Virtual. 2009. Recuperado de: http://www.bibliodrogas.cl/bibliodrogas/boletines/BOLETIN\%20 ENERO\%202009.pdf)

8. Robertson P, Hellriegel ET. Clinical pharmacokinetic profile of modafinil. Clin Pharmacokinet. 2003; 42: 123-137. doi. org/10.2165/00003088-200342020-00002

9. Volkow N, Fowler J, Logan J., et al. Effects of modafinil on dopamine and dopamine transporters in the male human brain: Clinical Implications. JAMA. 2009; 301: 1148- 1154.

10. Funayama T, Ikeda Y, Suzuki H. Modafinil augments brain activation associated with reward anticipation in the nucleus accumbens. Psychopharmacology. 2014; 231: 3217-28. doi:10.1007/s00213-014-3499-0.

11. Ballon J, Feifel D. A systematic review of modafinil: Potencial clinical uses and mechanism of action. J Clin Psychiatry 2006; 67: $554-566$.

12. Minzenberg M, Carter C. Modafinil: A review of neurochemical actions and effects on cognition. Neuropsychopharmacology 2008; 33: 1477-1502. doi: 10.1038/sj.npp.1301534

13. Moher D, Liberati A, Tetzlaff J, Altman DG, PRISMA Group. Preferred reporting items for systematic reviews and metaanalyses: the PRISMA statement. PLoS Med. 2009; 6. doi: 10.1371/journal.pmed.1000097.

14. Biederman J, Swanson J. M, Wigal S, Kratochvil C. J, Boellner S. W, Earl C. Q, Jiang J, Greenhill L. Efficacy and safety of modafinil film-coated tablets in children and adolescents with attentiondeficit/hyperactivity disorder: results of a randomized, doubleblind, placebo-controlled, flexible-dose study. Pediatrics. 2005; 116: 777-784. doi: 10.1542/peds.2005-0617

15. Roth T, Schwartz J. R, Hirshkowitz M, Erman M. K, Dayno J. M, Arora S. Evaluation of the Safety of Modafinil for Treatment of Excessive Sleepiness. J Clin Sleep Med. 2007; 3: 595-602.

16. Spiller H. A, Borys D, Griffith J, Klein-Schwartz W, Aleguas A, Sollee D, Anderson D. A, Sawyer T. S. Toxicity from modafinil ingestion. Clinical Toxicology. 2009; 47: 153-156. doi:10.1080/15563650802175595

17. Olivares J, González-Hernández J. Eficacia del modafinilo como potenciador cognitivo en sujetos sanos. Rev. Memoriza.com. 2009; 2: 39-43.

18. Aytas Ö, Dílek H. Modafinil-induced psychosis: A case report. Noro Psikiyatr Ars. 2015; 52: 99-101. doi: 10.5152/npa.2015.7160

19. Younes D, Asch F. Tako-Tsubo Cardiomyopathy Following Modafinil Use. J Clin Pharmacol. 2013; 53: 662-664. doi: 10.1002/ jcph.77

20. Binnenmars H, Idzerda H, Tan H, Linssen G. Ventricular tachycardia during treatment with modafinil for narcolepsy: a 
case report. International Journal of Clinical Medicine. 2012; 3 : 513-517. doi: 10.4236/ijcm.2012.36092

21. Amariles, P. A comprehensive literature search: drugs as possibletriggers of takotsubo cardiomyopathy. Curr Clin Pharmacol. 2011; 6: 1-11. doi: 10.2174/157488411794941340

22. Kpaeyeh, J, Mar P, Raj V, Black B, Arnold A, Biaggioni I, Shibao C, Paranjape S, Dupont W, Robertson D, Raj S. Hemodynamic Profiles and Tolerability of Modafinil in the Treatment of POTS a randomized placebo-controlled trial. J Clin Psychopharmacol. 2014; 34: 738-741. doi: 10.1097/JCP.0000000000000221

23. Taneja I, Diedrich A, Black B, Byrne D, Paranjape S, Robertson D. Modafinil elicits sympathomedullary activation. Hypertension. 2005; 45: 612-618. doi:10.1161/01.hyp.0000158267.66763

24. Rattray B, Martin K, Hewitt A, Cooper G, McDonald W. Effect of acute modafinil ingestion on cognitive an physical performance folloeing mental exertion. Hum Psychopharmacol Clin Exp. 2019; 34: e2700.

25. Kaplan S, Goehring E, Melamed-Gal S, Nguyen-Khoa B, Knebel $\mathrm{H}$, Jones J. Modafinil and the risk of cardiovascular events: findings from three US claims databases. Pharmacoepidemiol Drug Saf. 2018; 27: 1-9. doi: 10.1002/pds.4642

26. Mo Y, Thomas M, Miano T, Stemp L, Bonacum J, Hutchins K, Karras G. Effect of Modafinil on cognitive function in intensive care unit patients: A retrospective cohort study. The Journal of Clinical Pharmacology. 2018; 58: 152-157. doi: 10.1002/jcph.1002

27. Scammell T, Estabrooke I, McCarthy M, Chemelli R, Yanagisawa M, Miller M, Saper C. Hypothalamic Arousal Regions Are Activated during Modafinil Induced Wakefulness. J Neurosci. 2000; 20: 8620-8628. https://doi.org/10.1523/ JNEUROSCI.20-22-08620.2000

28. Gallopin T, Luppi P, Rambert F, Frydman A, Fort P. Effect of the Wake-Promoting Agent Modafinil on Sleep-Promoting Neurons from the Ventrolateral Preoptic Nucleus: an In Vitro Pharmacologic Study. Sleep. 2004; 27: 19-25.

29. Urbano F, Leznik E, Llinás R. Modafinil enhances thalamocortical activity by increasing neuronal electrotonic coupling. Proc Natl Acad Sci U S A. 2007; 104: 12554-12559. doi: 10.1073/ pnas.0705087104

30. Webb I, Pollock M, Mistlberger R. Modafinil [2-[(Diphenylmethyl) sulfinyl]acetamide] and Circadian Rhythms in Syrian Hamsters: Assessment of the Chronobiotic Potential of a Novel Alerting Compound. J Pharmacol Exp Ther. 2006; 317: 882-889. doi:10.1124/jpet.105.099010

31. Volkow N, Fowler J, Logan J, Alexoff D, Zhu W, Telang F, Wang G, Jayne M, Hooker J, Wong C, Hubbard B, Carter P, Warner D, King P, Shea C, Xu Y, Muench L, Apelskog-Torres K. Effects of Modafinil on Dopamine and Dopamine Transporters in the Male Human Brain. JAMA. 2009; 301: 1148-1154. doi: 10.1001/ jama.2009.351

32. Wisor J. Modafinil as a catecholaminergic agent: empirical evidence and unanswered questions. Front Neurol. 2013; 4: 139. doi: 10.3389/fneur.2013.00139

33. Mereu M, Chun L, Prisinzano T, Newman A, Katz J, Tanda G. The Unique Psychostimulant Profile of $( \pm)$-Modafinil: Investigation of Behavioral and Neurochemical Effects in Mice. 2017; 45: 167174. doi: 10.1111/ejn.13376

34. Burrows B, Watterson L, Johnson M, Olive M. Effects of Modafinil and R-Modafinil on Brain Stimulation Reward Thresholds: Implications for Their Use in the Treatment of Psychostimulant Dependence. J Drug Alcohol Res. 2015; 4: 235958. doi: 10.4303/ jdar/235958

35. Dias V, Rosa H, D'avila L, Vey L, Barcelos R, Burger M. Modafinil reduces amphetamine preference and prevents anxiety-like symptoms during drug withdrawal in young rats: Involvement of dopaminergic targets in VTA and striatum. Prog Neuropsychopharmacol Biol Psychiatry. 2019; 92: 199-206. doi: 10.1016/j.pnpbp.2019.01.007

36. Karabacak Y, Sase S, Aher Y, Sase A, Saroja S, Cicvaric A, Höger
H, Berger M, Bakulev V, Sitte H, Leban J, Monje F, Lubec G. The effect of modafinil on the rat dopamine transporter and dopamine receptors D1-D3 paralleling cognitive enhancement in the radial arm maze. Front Behav Neurosci. 2015; 9: 215. doi: 10.3389/fnbeh.2015.00215

37. Fernández A, Mascayano F, Lips W, Painel A, Norambuena J, Madrid E. Effects of modafinil on attention performance, shortterm memory and executive function in university students. A randomized trial. Medwave. 2015; 15: 6166. doi: 10.5867/ medwave.2015.05.6166

38. Bellebaum C, Kuchinke L, Roser P. Modafinil alters decision making based on feedback history - a randomized placebocontrolled double blind study in human. J Psychopharmacol. 2017; 31: 243-249. doi: 10.1177/0269881116668591

39. Turner D, Robbins T W, Clark L, Aron A R, Dowson J, Sahakian B J. Cognitive enhancing effects of modafinil in healthy volunteers. Psychopharmacology. 2003; 165: 260-269. doi 10.1007/s00213002-1250-8

40. Punzi M, Gili T, Petrosini L, Caltagirone C, Spalletta G, Sensi $\mathrm{S}$ L. Modafinil-induced changes in functional connectivity in the cortex and cerebellum of healthy elderly subjects. Frontiers in aging neuroscience. 2017; 9: 1-9. doi: 10.3389/ fnagi.2017.00085

41. Ghahremani D G, Tabibnia G, Monterosso J, Hellemann G, Poldrack R A, London E D. Effect of modafinil on learning and task-related brain activity in methamphetamine-dependent and healthy individuals. Neuropsychopharmacology. 2011; 36: 950959. DOI:10.1038/npp.2010.233

42. Del Percio C, Derambure P, Noce G, Lizio R, Bartrés D, Blin O, Payoux P, et al. Sleep deprivation and nodafinil effect cortical sources of resting state electroencephalographic rhythms in healthy young adults. Clinical Neurophysiology. 2019; 130 : 1488-1498.

43. Müller U, Rowe J B, Rittman T, Lewis C, Robbins T W, Sahakian $B$ J. Effects of modafinil on non-verbal cognition, task enjoymen and creative thinking in healthy volunteers. Neuropharmacology. 2013; 64: 490-495.

44. Gilleen J, Michalopoulou P G, Reichenberg A, Drake R, Wykes T, Lewis S W, Kapur S. Modafinil combined with cognitive training is associated with improved learning in healthy volunteers- A randomised controlled trial. European Neuropsychopharmacology. 2014; 24: 529-539.

45. Repantis D, Schlattmann P, Laisney O, et al. Modafinil and methylphenidate for neuroanhancement in healthy individuals: A systematic review. Pharmacological Research 2010; 62: 187-206.

46. Lees J, Michalopoulou P G, Lewis S W, Preston S, Bamford C, Collier T, Kalpakidou A, Wykes T, Emsley R, Pandina G, Kapur S, Drake R J. Modafinil and cognitive enhancement in schizophrenia and healthy volunteers: the effects of test battery in a randomized controlled trial. Psychological Medicine. 2017; 47: 2358-2368.

47. Battleday R M, Brem A K. Modafinil for cognitive neuroenhancement in healthy non-sleep-deprived subjects: A systematic review. European Neuropsychopharmacology. 2015; 25: 1865-1881.

48. Yamada Y, Inagawa T, Sueyoshi K, Sugawara N, Ueda N, Omachi Y, et al. Social cognition deficits as a target of early intervention for psychoses: a systematic review. Frontiers of Psychiatry. 2019; 10: 333.

49. Kaser M, Deakin J B, Michael A, Zapata C, Bansal R, Ryan D, Cormack F, Rowe J B, Sahakian B J. Modafinil improves episodic memory and working memory cognition in patients with remitted depression: a double-blind, randomized, placebo-controlled study. Biological Psychiatry: Cognitive Neuroscienceand Neuroimaging. 2017; 2: 115-122.

50. Ojeda N., Del Pino R., Ibarretxe-Bilbao N., Schretlen D. J., \& Pena J. (2016). [Montreal Cognitive Assessment Test: Normalization and standardization for Spanish population]. Revista de Neurologia, 63, 488-496. [PubMed] 